\title{
The Importance of Spatiotemporal Fish Population Dynamics in Barrier Mitigation Planning
}

\author{
Christina Ioannidou Jesse R. O’Hanley* \\ Kent Business School, University of Kent, Canterbury CT2 7FS, United Kingdom
}

\begin{abstract}
In this study, we propose a novel framework combining spatially explicit population viability analysis and optimization for prioritizing fish passage barrier mitigation decisions. Our model aims to maximize the equilibrium population size, or alternatively minimize the extinction risk, of a target fish species subject to a budget on the total cost of barrier mitigation. A case study involving a wild coho salmon (Oncorhynchus kisutch) population from the Tillamook basin, Oregon, USA is used to illustrate the benefits of our approach. We consider two different spawning adult dispersal patterns, river and reach level homing, as well as straying. Under density dependent population growth, we find that homing behavior type has a significant effect on barrier mitigation decisions. In particular, with reach homing, our model produces virtually the same population sizes as a more traditional barrier prioritization procedure designed to maximize accessible habitat. With river homing, however, we find that it is not necessary to remove all barriers in order to maximize equilibrium population size. Indeed, a stochastic version of our model reveals that removing all barriers actually results in a marginal increase in quasi-extinction risk. We hypothesize that this is due to a population thinning effect of barriers, resulting in a surplus of recruits in areas of low spawner density. Our findings highlights the importance of considering spatiotemporal fish population dynamics in river connectivity restoration planning. By adding greater biological realism, models such as ours can help conservation managers to more strategically allocate limited resources, resulting in both cost savings and improved population status for a focal species.
\end{abstract}

Keywords: river connectivity; optimization; population viability analysis; density dependence; homing; coho salmon.

\footnotetext{
*Correspondence email: $\mathrm{j}$. ohanley@kent.ac.uk
} 


\section{Introduction}

Dams, culverts, and other artificial in-stream structures can hinder or even entirely block migratory fish from reaching essential breading and rearing habitats (Januchowski-Hartley et al., 2013). The end result is the restricted range size, reduced productivity, and genetic isolation of migratory fish populations, as well as wider changes in aquatic community composition (Stanford et al., 1996; Bednarek, 2001; Lucas and Baras, 2001). Reconnecting stream habitats isolated by the presence of so called fish passage barriers is widely considered a top priority for restoring healthy freshwater ecosystems (Roni et al., 2008).

In this paper, we present a framework for prioritizing barrier mitigation in connectivity impaired river networks to improve the viability of a diadromous fish population. In particular, we integrate spatially explicit population viability analysis (PVA) into a heuristic optimization model with the aim of maximizing equilibrium population size (deterministic version) or minimizing extinction risk (stochastic version) for a target species. A case study involving a wild coho salmon (Oncorhynchus kisutch) population from the Tillamook basin along the Oregon Coast, USA is used to illustrate the utility of our approach.

PVA models have seen extensive use in the context of fisheries and river habitat management. Scheuerell et al. (2006), for example, propose a PVA model to evaluate the effects of habitat change, hatchery operations, and harvest management actions on salmon population status. They use a multistage Beverton-Holt model to describe the production of salmon from one life stage to the next and to provide estimates of abundance, productivity, spatial structure, and diversity. Their model is used to evaluate the potential consequences of habitat conservation alternatives in Snohomish River basin in Washington State. Nickelson and Lawson (1998), meanwhile, develop a life-cycle model to estimate the extinction probability of coho salmon populations along the Oregon Coast. Spawner abundance, demographic and environmental stochasticity, genetic effects, density, and habitat driven survival rates are all taken into account. A comprehensive review on the use of PVA models in the planning of recovery actions for Atlantic and Pacific salmon can be found in Sweka and Wainwright (2014).

PVA studies focusing on the importance of river connectivity for aquatic species persistence are few in number and often limited to experiments with virtual populations. Nieland et al. (2015), one of the few dealing with a real population, examine mortality impacts of large hydropower dams on Atlantic salmon in the Penobscot River, Maine. Their model, which tracks both the number and location of fish at multiple life stages, is used to evaluate relative changes in abundance of six dam removal scenarios. Labonne et al. (2008) explore the relationship between connectivity in dendritic networks and population dynamics. The authors find that species with low dispersal ability benefit from higher connectivity, in terms of reduced local extinction 
and genetic isolation, but that for high dispersal species, metapopulation size is negatively correlated with connectivity. Another good example is Harvey and Railsback (2012), who analyze the effects of fish passage barriers on a virtual resident trout population. A detailed individual-based model is developed to capture the demographics and fine-scale movements of trout. Simulations with varying barrier densities are analyzed to investigate how the location of barriers affect two population stability properties: persistence and resistance. Interestingly, they find that low barrier densities can actually produce an increase in overall biomass.

Research into prioritizing the repair/removal of fish passage barriers has progressed mostly in parallel with the development of PVA for freshwater fisheries conservation. Traditionally, prioritization has relied on the use of scoring-and-ranking type procedures (e.g., Kocovsky et al., 2009). However, because barriers are evaluated in isolation, thus ignoring the interactive effects of multiple repair/removal actions on river connectivity, scoring-and-ranking has been shown to produce highly inefficient solutions (O'Hanley and Tomberlin, 2005). To overcome this deficit, Kemp and O'Hanley (2010) recommend the use of spatially informed graph theory models (e.g., Eros et al., 2011) and optimization based approaches (e.g., King et al., 2017). Unlike graph theory, optimization provides prescriptive solutions that maximize gains from river connectivity restoration given limited resources (King and O'Hanley, 2016).

A range of barrier optimization methods have appeared in recent years. The vast majority of these are built around relatively simple habitat connectivity indices (Kuby et al., 2005; O'Hanley, 2011; O'Hanley et al., 2013; Neeson et al., 2015), which only consider dispersal at an abstract level. Rarely have complex fish dispersal and population growth been treated in comprehensive manner. Work by Paulsen and Wernstedt (1995), Newbold and Siikamaki (2009), Zheng and Hobbs (2013), and Fitzpatrick and Neeson (2018) provide notable examples. Paulsen and Wernstedt (1995) propose a combined simulation-optimization methodology to analyze the cost-effectiveness of different mitigation measures for restoring salmon populations in the Columbia River basin, including in-stream habitat restoration and improved fish passage past large hydropower dams and small barriers. The simulation model is used to evaluate the biological effects of a specified combination of mitigation actions, while the optimization model finds the least-cost portfolio of mitigation actions that achieves stock-specific harvesting and escapement goals. As pointed out in Kemp and O'Hanley (2010), this approach is restricted to dealing with a rather small number of potential alternatives, as every feasible combination of mitigation actions must be individually simulated. Zheng and Hobbs (2013) employ a multiobjective approach for optimizing dam removal decisions in the Lake Erie basin. A number of ecological and socioeconomic criteria are considered, including native fish abundance, recreation/commercial harvesting, dam safety, and dam removal and invasive species management costs. A complex, ecosystem simulation model is used to link dam removal decisions to lake-wide ecological effects. Importantly, the authors make 
the strong simplifying assumption that fish recruitment and fish community structure respond linearly to increases in habitat availability. This greatly reduces computational overhead but also limits the generality of their approach. Finally, Newbold and Siikamaki (2009) develop a PVA model and incorporate it into a reserve site selection (RSS) algorithm to conserve Columbia River salmon populations. The PVA model is used to estimate how watershed conservation, consisting of a suite of land-use management activities that reduce non-point source pollution within a watershed, affect the long-term probability of persistence of salmon. The RSS algorithm is used to select different watersheds for conservation based on their cost-effectiveness in increasing persistence. Options for barrier repair/removal are not considered.

The aim of our present work is to address various shortcomings of existing barrier prioritization methods, which include: i) the use of structural (i.e., habitat connectivity) indices as proxies for complex biological processes (i.e., fish dispersal and population growth); ii) overly restrictive assumptions that weaken generality (e.g., all response functions must be linear); and iii) limited scalability that prevents evaluating a large number of barriers and mitigation options (e.g., having to enumerate all feasible solutions). To our knowledge, ours is the first attempt at directly incorporating spatial PVA into a barrier optimization model, as opposed to loosely coupling optimization with PVA (sensu Paulsen and Wernstedt, 1995; Zheng and Hobbs, 2013). The remainder of the paper is organized as follows. We start by outlining our optimization framework, which includes a formal mathematical formulation of the problem and the basic steps involved with deriving estimates of population viability. This is followed by a description of different plausible dispersal patterns for spawning adult coho salmon, how population growth dynamics (deterministic and stochastic) are modeled, the solution algorithm used to solve our model, and basic data processing requirements. Results from our case study are then presented along with a comparison our proposed method to a standard barrier optimization approach that maximizes accessible habitat. We end with some discussion points and suggestions for future research.

\section{Materials and Methods}

\subsection{Barrier Optimization Model}

In what follows, we propose an optimization model for cost-effectively targeting the mitigation (i.e., removal, replacement, or retrofitting) of in-stream barriers that negatively impact river connectivity. Two different variants of the model are considered, depending on how population growth is modeled. The deterministic version, referred to as MaxPop, seeks to maximize the equilibrium population size for a given target species via increased dispersal and habitat utilization. The stochastic version, referred to as MinExP, attempts to 
minimize the probability of population extinction over a given time horizon. In our current application, we restrict ourselves to fish species with a diadromous life-cycle, focusing specifically on salmon.

We make the following assumptions. The river network under consideration is strictly "dendritic," meaning it never diverges in the downstream direction, thus excluding braided river systems. Given this assumption, there is always a unique path from the river mouth to any point upstream. We further assume that each barrier has a known passability value. Passability refers to the fraction of fish, in the range $0-1$, that can successfully pass a barrier (Kemp and O'Hanley, 2010). Passability is normally species, life-stage (e.g., adult versus juvenile), and directionally (i.e., upstream versus downstream) dependent. Cumulative passability, in turn, refers to the combined effect that barriers have on fish dispersal. Assuming that barrier passabilities are independent, cumulative passability is calculated by multiplying the passabilities of all barriers along the path from a given origin (e.g., the ocean) to a given destination (e.g., an upstream spawning area). Barrier mitigation is carried out to increase the upstream and/or downstream passability of a barrier for one or more life-stages. For any particular barrier, there may be multiple mitigation options available. Finally, the total cost of mitigation must not exceed a specified budget.

Our model is formulated as follows. Let $J$, indexed by $j$, be the set of physical barriers, both artificial and natural, within a river network, and let $J^{\prime}$ be the subset of artificial barriers. The set of barrier mitigation actions available at artificial barrier $j$ is denoted by $A_{j}$. The cost of implementing mitigation option $i$ at barrier $j$ is given by $c_{j i}$. The total barrier mitigation budget is denoted by $b$. Decision variable $x_{j i}$ equals 1 if mitigation option $i$ is selected for barrier $j, 0$ otherwise. Given a vector of barrier mitigation decisions $\mathbf{x}$, function $F(\mathbf{x})$ expresses the population metric of interest - equilibrium population size (e.g., number of breeding adults) for MaxPop, probability of extinction (e.g., over the next 100 generations) for MinExP taking into account dispersal behavior (possibly life-stage specific), level of river connectivity, and population growth dynamics.

$$
\begin{aligned}
& \max _{\mathbf{x}} F(\mathbf{x}) \text { or } \min _{\mathbf{x}} F(\mathbf{x}) \\
& \text { s.t. } \\
& \quad \sum_{j \in J^{\prime}} \sum_{i \in A_{j}} c_{j i} x_{j i} \leq b \\
& \qquad \sum_{i \in A_{j}} x_{j i} \leq 1 \quad \forall j \in J^{\prime} \\
& \quad x_{j i} \in\{0,1\} \quad \forall j \in J^{\prime}, i \in A_{j}
\end{aligned}
$$




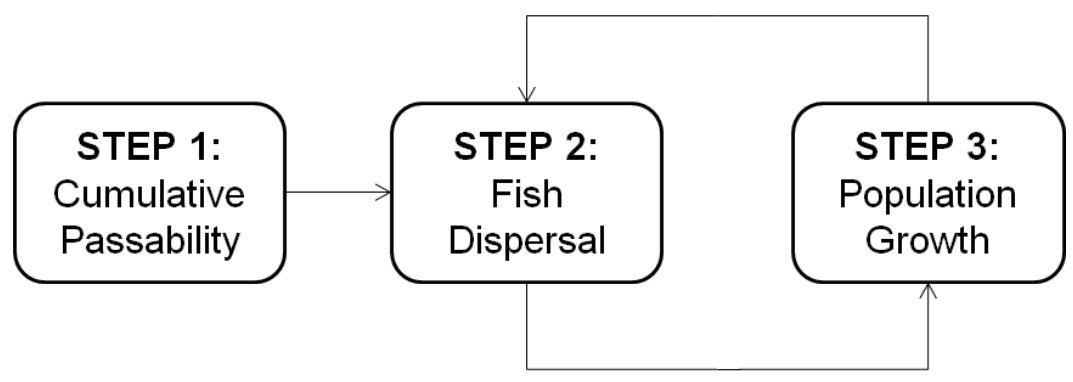

Figure 1: Evaluation steps for determining equilibrium population size.

The objective (1) either maximizes equilibrium population size (MaxPop) or minimizes the probability of extinction (MinExP). Constraint the(2) requires that the total cost of barrier mitigation actions not exceed the available budget. Constraints (3) ensure that at most one mitigation project can be carried out at each artificial barrier. Finally, constraints (4) impose binary restrictions on the barrier mitigation decision variables.

The steps involved in determining population metric $F(\mathbf{x})$ (i.e., equilibrium population size or extinction probability) for barrier mitigation solution $\mathbf{x}$ are outlined in Figure 1. In Step 1, cumulative passability to spawning/rearing areas is determined based on the barrier passabilities produced by solution $\mathbf{x}$. For spawning adult salmon, cumulative passability $\alpha_{j}$ to spawning areas immediately above barrier $j$ would be calculated as $\alpha_{j}=\prod_{k \epsilon D_{j}}\left(p_{k}^{0}+\sum_{i \in A_{j}} p_{j i}^{\prime} x_{j i}\right)$, where $D_{j}$ is the subset of barriers downstream from and including barrier $j, p_{j}^{0}$ is the initial passability of barrier $j$, and $p_{j i}^{\prime}$ is the increase in passability given implementation of mitigation option $i$ at barrier $j$. In Step 2, fish dispersal to spawning/rearing occurs according to assumed adult/juvenile dispersal patterns and level of longitudinal connectivity. Population growth takes place in Step 3 , with the number of recruits being produced in a particular habitat area possibly density dependent. Note that Steps 2 and 3 can be formed of multiple sub-steps if dispersal and productivity/survival are life-stage dependent. Steps 2 and 3 need to be repeated iteratively from one generation to the next until equilibrium population size is achieved or the population goes extinction. Depending on the type of homing pattern, fish dispersal (Step 2) may need to be recalculated for each generation. Note that each barrier mitigation solution $\mathbf{x}$ can produce a different equilibrium population size or extinction probability estimate, which means that the whole process needs to be repeated any time a new barrier mitigation solution is evaluated.

In what follows, the various homing patterns considered in our study are discussed in Section 2.2. In Section 2.3, we cover fish population growth dynamics. Our solution methodology is presented in Section 2.4. Finally, in Section 2.5 we provide background information about our study area and the input data used to parameterize our model. 


\subsection{Dispersal Patterns}

Upstream migrating adult salmon usually return (i.e., home) to their natal rivers to spawn. Salmon homing can be conceptualized along a hierarchy of spatial scales (Quinn, 1997), starting with the watershed, then main tributary, followed by stream reach, and finally down to a specific point within a stream reach (i.e., the redd). Naturally, homing is more accurate at broader spatial scales. Often a small percentage of adult fish, referred to as strays, move into non-natal streams during upstream movement, which has implications for metapopulation persistence. In this study, two different homing patterns (at either end of the site homing continuum) are considered: river and reach homing. For river homing, it is assumed that adults have low homing fidelity. After returning to their natal river, adults disperse freely within the river to find suitable spawning habitat. For reach homing, it is assumed that adults have much higher homing fidelity and will attempt to return to their specific natal stream reach. These two dispersal patterns are discussed in detail below. For simplicity, juvenile fish are assumed to have suitable rearing habitat within the vicinity of the spawning area from which they emerge and so do not make appreciably long distance dispersal movements to rearing areas upstream or downstream.

\subsubsection{River Homing}

With river homing, adult salmon are assumed to distribute within their natal river according to an ideal free distribution (IFD, Case, 1999). Under IFD, "consumers" (i.e., fish), have full knowledge of habitat resources and disperse in such a way that consumer density is uniform. For our study, habitat resources are assumed to be proportional to river length. If no barriers were present, the number of spawners per unit length of river would be the same in each spawning area. With barriers present, however, dispersal is disrupted and equal densities may not be achieved.

To model the effect that barriers have on driving spawner densities away from ideal, we develop a linear program referred to as the Ideal Free Distribution with Barriers Problem (IFDBP). The model seeks to minimize the maximum difference between the ideal spawner density and what can be achieved given the presence of barriers. In addition to the notation introduced previously, let $p_{j}$ be the current passability of barrier $j$. Following King and O'Hanley (2016), the section of river above a barrier up to the next set of barriers or the river terminus is referred to as a river subnetwork. The amount of spawning habitat in subnetwork $j$ is given by $v_{j}$, while the total amount of spawning habitat within the entire watershed is denoted by $V$ (i.e., $V=\sum_{j \epsilon J} v_{j}$ ). The total number of spawners is denoted by $N$. Finally, consider the 
following decision variables.

$$
\begin{aligned}
& y_{j}=\text { number of spawners dispersing to subnetwork } j \\
& z=\text { maximum difference between observed and ideal spawner density for any subnetwork }
\end{aligned}
$$

With this in place, a mathematical formulation of IFDBP is given below.

$\min z$

s.t.

$$
\begin{aligned}
& \sum_{j \in J} y_{j}=N \\
& y_{j} \leq \prod_{k \in D_{j}} p_{k} N \quad \forall j \in J \\
& z \geq \frac{1}{v_{j}} y_{j}-\frac{1}{V} N \quad \forall j \in J \mid v_{j}>0 \\
& y_{j}=0 \quad \forall j \in J \mid v_{j}=0 \\
& y_{j} \geq 0 \quad \forall j \in J
\end{aligned}
$$

The objective function (5) minimizes the maximum deviation in spawner density away from ideal across all river subnetworks. The first constraint (6) forces the sum of the spawners across all subnetworks $\sum_{j \epsilon J} y_{j}$ to be equal to the total number of spawners $N$. Constraints (7) restrict $y_{j}$ not to exceed the total number of fish that can potentially reach subnetwork $j$, which is equal to the cumulative passability $\prod_{k \in D_{j}} p_{k}$ of barrier $j$ times the number of spawners $N$. Constraints (8) specify for all subnetworks $j$ with non-zero amounts of spawning habitat $\left(v_{j}>0\right)$ that the maximum difference in spawner density $z$ from ideal must be greater than or equal to the observed density $y_{j} / v_{j}$ in subnetwork $j$ minus the ideal density $N / V$. Inequalities (9) force $y_{j}$ to be zero for all subnetworks with zero spawning habitat, since no fish would migrate to such areas. Lastly, inequalities (10) impose non-negativity restrictions on the $y_{j}$ decision variables.

Note that when evaluating a fish metapopulation that distributes among multiple rivers, model IFDBP needs to be solved separately for each river network. Before doing so, the number of outgoing strays and the number of incoming strays needs to be accounted for in order to specify the correct spawner population sizes $N$ that will distribute within each river network. Further note that as long as cumulative passability values do not 
change (i.e., no additional mitigation is carried out) then the relative proportions of fish migrating to any particular river subnetwork will remain constant even if the total spawner population size $N$ subsequently changes in later generations. The importance of this is that IFDBP only needs to be solved once when determining equilibrium population size.

\subsubsection{Reach Homing}

The second adult dispersal pattern examined in this study is reach homing. Here, fish are expected to return to the natal stream reach from which they emerged, which for our purposes is taken to be their originating river subnetwork. If no barriers were present, then any fish spawned in subnetwork $j$ would be able to return to $j$ as adults. When barriers are present, however, of the number of fish $N^{j}$ spawned in subnetwork $j$, only a fraction, equal to $N^{j}$ times the cumulative passability $\prod_{k \epsilon D_{j}} p_{k}$ of barrier $j$, will be able to do so. The rest will be "trapped" in the subnetworks downstream of $j$.

Consequently, with reach homing, the number of spawners $y_{j}$ contained in subnetwork $j$ is calculated as the sum of the spawners that originated there and successfully returned plus a portion of spawners that were unsuccessful in reaching subnetworks further upstream due to passability restrictions. In addition, the number of spawners that end up in subnetwork $j$ is affected by fish straying. In particular, a small percentage of fish spawned in $j$ will stray away from $j$ to other subnetworks and a small number of fish will be redirected to subnetwork $j$ after straying from other reaches. For simplicity, we assumed a fixed percentage of fish would stray from any subnetwork and then redistribute themselves by spreading equally among watersheds and then with equal probability among subnetworks within a watershed. Unlike with river homing, fish dispersal calculations need to be updated at every generation for reach level homing.

\subsection{Population Growth}

\subsubsection{Deterministic}

We estimate density dependent salmon recruitment using the well-known Ricker model. According to the Ricker model, the expected number of recruits $N_{t+1}$ in generation $t+1$ is determined by the equation:

$$
N_{t+1}=N_{t} e^{r\left(1-\frac{N_{t}}{K}\right)}
$$

where $N_{t}$ is the number of spawners in generation $t, r$ is the intrinsic growth rate, and $K$ is the carrying capacity of the habitat. If $r$ is between 0 and 2 (i.e., $0<r<2$ ), the model has a stable equilibrium. Cycles or chaotic dynamics are produced for growth rates $r \geq 2$. 
In our implementation, the Ricker model was used to generate recruits $N_{t+1}^{j}$ separately for each subnetwork $j$ over 200 generations starting with an initial population size of $N_{0}^{j}$ in subnetwork $j$. At every generation, recruits are assumed to travel back to the sea and then disperse upstream according to either a river homing or reach homing dispersal pattern. The mean of the last 100 generations was used to compute the "equilibrium" population size, which could be stable, cyclic, or chaotic.

\subsubsection{Stochastic}

To account for the effects of environmental stochasticity on fish population growth, equation (11) can be replaced by the following:

$$
N_{t+1}=N_{t} e^{r\left(1-\frac{N_{t}}{K}\right)+\varepsilon_{t}}
$$

Parameter $\varepsilon_{t}$, which adjusts the nominal growth rate $r\left(1-N_{t} / K\right)$ up or down, is drawn from a normal distribution with a mean of zero and a variance of $n V_{r} /(n-1)$, where $n$ is the number of data points used in the linear regression analysis to derive Ricker model parameters $r$ and $K$ and $V_{r}$ is the residual variance (Morris and Doak, 2002). To generate estimates for the probability of extinction, population sizes were simulated across 50 generations using equation (12) and the fraction of simulation runs (out of 1000) in which population abundance fell below a quasi-extinction threshold (QET) was determined.

\subsection{Solution Methodology}

A flow chart of the heuristic algorithm used to solve MaxPop is shown in Figure 2. In Step 1, an initial starting solution is generated by solving an optimization model, referred to as MaxHab, which maximizes the amount of accessible (i.e., connectivity-weighted) habitat available to upstream migrating fish (King and O'Hanley, 2016). The equilibrium population size of this starting solution is computed (taking into account dispersal and population dynamics) and then accepted as the current best (aka incumbent) solution.

In Step 2, a local search is performed in an attempt to find a solution with higher equilibrium population size. First, a currently mitigated barrier is selected and its passability and the passabilities of all other mitigated barriers upstream from the selected barrier are temporarily reset to their initial passability values. The resulting cost savings from undoing mitigation for the selected barrier and those upstream is added back to the remaining budget and the equilibrium population size is recalculated. A new candidate solution is then constructed using a "greedy" add procedure, whereby the barrier mitigation option with the largest benefit-to-cost ratio (net change in equilibrium population size divided by cost) is selected one after another 


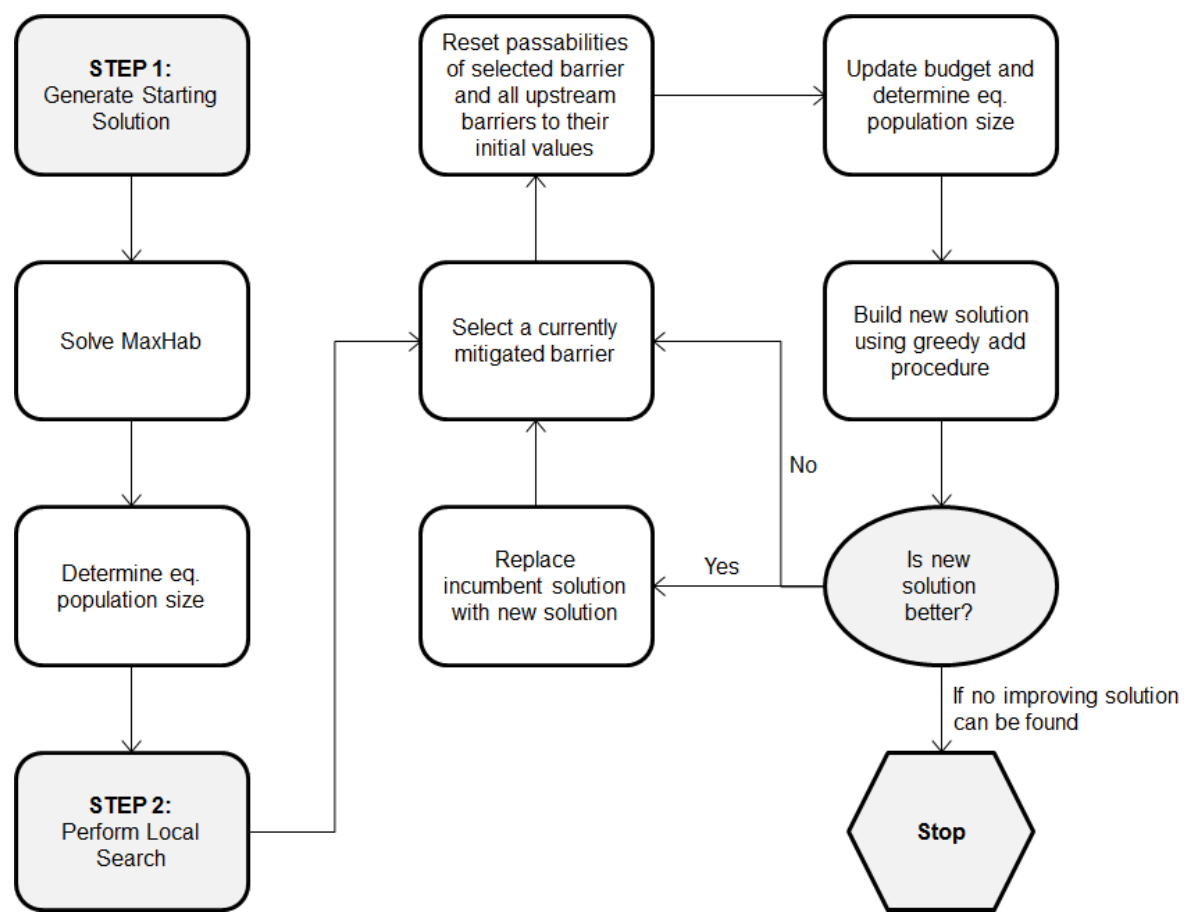

Figure 2: MaxPop solution algorithm.

until either the remaining budget is exhausted or no improvement in equilibrium population size can be achieved. Note that for applications involving a diadromous species like salmon, if zero-passability barriers are present downstream from a barrier being considered for mitigation, these should be mitigated at the same time. Intuitively, it would not make sense to mitigate such a barrier if impassable barriers downstream would block the upstream migration of diadromous fish. Once a proposed candidate solution has been built, it is then compared to the incumbent solution. If the candidate solution results in a higher equilibrium population size, it replaces the incumbent solution. Otherwise, the algorithm goes back to the incumbent solution and the above procedure is repeated for a different selected barrier.

MinExP was solved using the same basic heuristic method developed for MaxPop but with a few straightforward modifications. Specifically, we changed the objective to minimizing quasi-extinction and, in the case of river homing, used the solution produced by MaxPop as the initial starting solution instead of the MaxHab solution.

\subsection{Study Area}

To illustrate the benefits of our proposed modeling framework, we examined the impact of barrier removal on wild adult coho salmon (O. kisutch) in the Tillamook basin of Oregon, USA. Oregon Coast coho salmon are currently listed as a threatened species under the Endangered Species Act. Construction of fish passage 


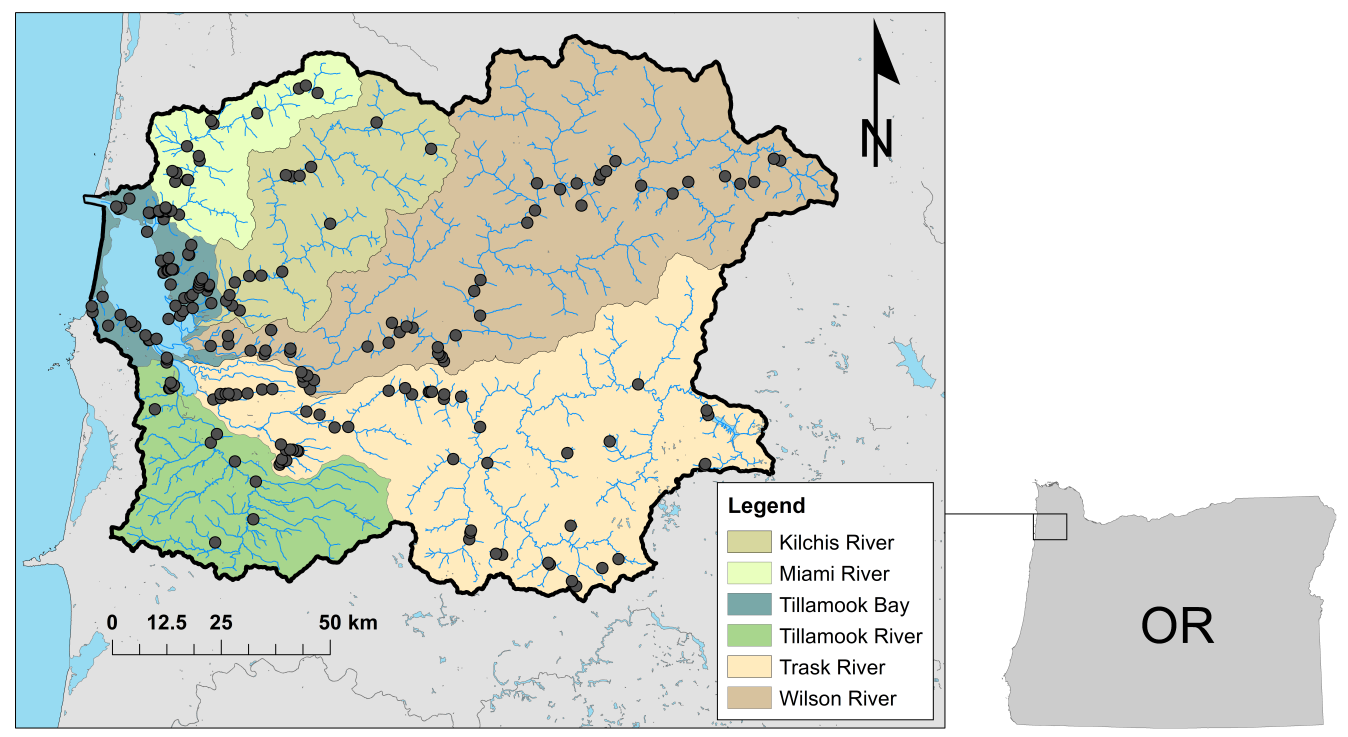

Figure 3: Watersheds and barrier locations (dark gray circles) in the Tillamook basin.

barriers up and down the coast has caused extensive loss of access to historical coho salmon habitats. Improved fish passage is considered essential to the successful recovery of Oregon Coast coho (NMFS, 2015).

Oregon Coast coho salmon typically spawn in small streams NOAA (2017). Working off a 1:100,000 scale river network layer created by the Oregon Department of Forestry, we treated all river segments of Strahler stream order 1 as potential spawning habitat. Strahler order of each river segment was determined using the RivEX toolbox for ArcGIS 10.2.1 (Hornby, 2014). In the absence of barrier mitigation, total accessible spawning habitat in the Tillamook basin is estimated to be $408 \mathrm{~km}$.

Data on the location of 202 culverts, dams, fords, and tidegates within the Tillamook basin were derived from Pilson (2012). Each barrier is georeferenced and includes a description of its structure type, available mitigation options, and estimated costs. In the absence of extensive field surveys or other relevant data for assessing barrier passability individually (for a review of barrier assessment methods see Kemp and O'Hanley,

Table 1: Initial passability values.

\begin{tabular}{lc}
\hline Barrier Type & Passability \\
\hline Culvert & 0.2 \\
Dam & 0 \\
Ford & 0.9 \\
Tidegate & 0.9 \\
Weir & 0 \\
Other & 0 \\
\hline
\end{tabular}




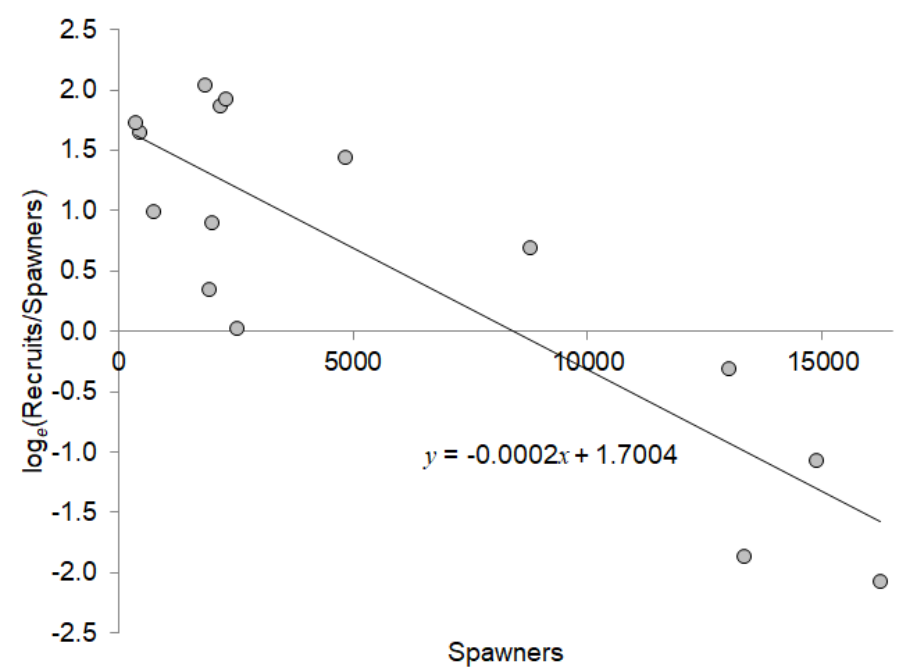

Figure 4: Estimated number of recruits per spawner ( $\log _{e}$ transformed) versus number of spawners (gray circles) for Tillamook basin wild coho (O. kisutch) and linear regression line (solid black line) for deriving Ricker model parameters (intercept: $r$; slope: $-r / K$ ).

2010), we assigned initial passability based on structure type as shown in Table 1 . We assumed that barriers were fully passable in the downstream direction (i.e., for outward smolt migration) that mitigation would restore upstream passability to a value of 1.0. Barrier points were snapped to the river network using a $50 \mathrm{~m}$ snapping distance. RivEX was then used to split the river network at barrier points and determine key barrier information, including a barrier's immediate downstream barrier (required to generate set $D_{j}$ ) and net length of upstream spawning habitat (model parameter $v_{j}$ ). In order to account for spawning habitat between the river mouth and the first set of artificial barriers, a "dummy" barrier, with initial passability equal to 1 and no mitigation option, was added at river mouths. Mouth nodes were identified using RivEX. After snapping, the final dataset consisted of 193 artificial barriers and 19 dummy barriers spread among 6 watersheds (the Miami, Kilchis, Tillamook, Trask, Wilson and Tillamook Bay watersheds), as shown in Figure 3.

Population counts and harvest rates for wild coho spawners in the Tillamook basin for the period 1996 to 2013 were obtained from the Oregon Adult Salmonid Inventory and Sampling Project (OASIS, 2016). Recruits were assumed to return as adults 3 years after emerging, with the number of recruits $R_{t}$ produced in year $t$ equal to $S_{t+3} /\left(1-h_{t+3}\right)$, where $S_{t+3}$ is the number of recorded spawners and $h_{t+3}$ is the harvest rate 3 years after $t$. A scatter plot of the number of recruits per spawner ( $\log _{e}$ transformed) versus number of spawners is shown in Figure 4. Ricker model parameters were estimated in the usual way by fitting a linear regression line to these points. The regression model, which had an adjusted $\mathrm{R}^{2}$ of 0.725 , produced estimates of $r=1.70$ and $K=8442$ (overall carrying capacity in the Tillamook basin). By comparison, the adjusted 


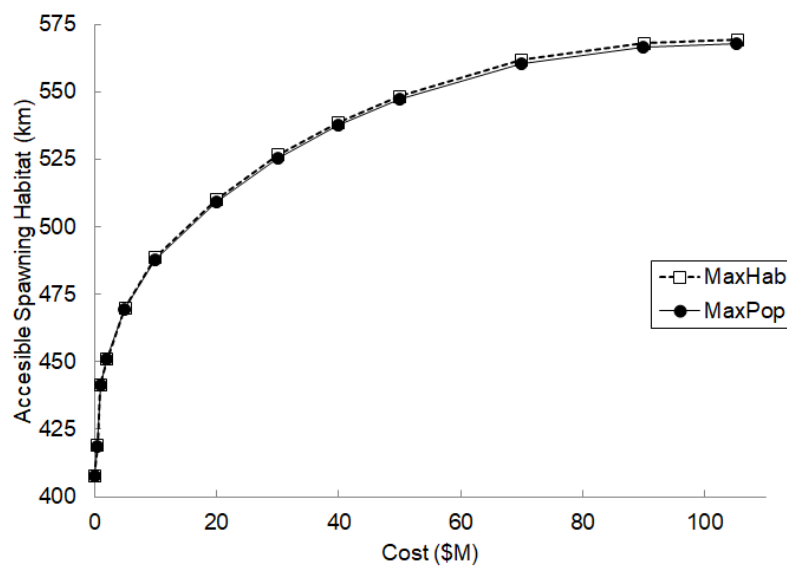

(a)

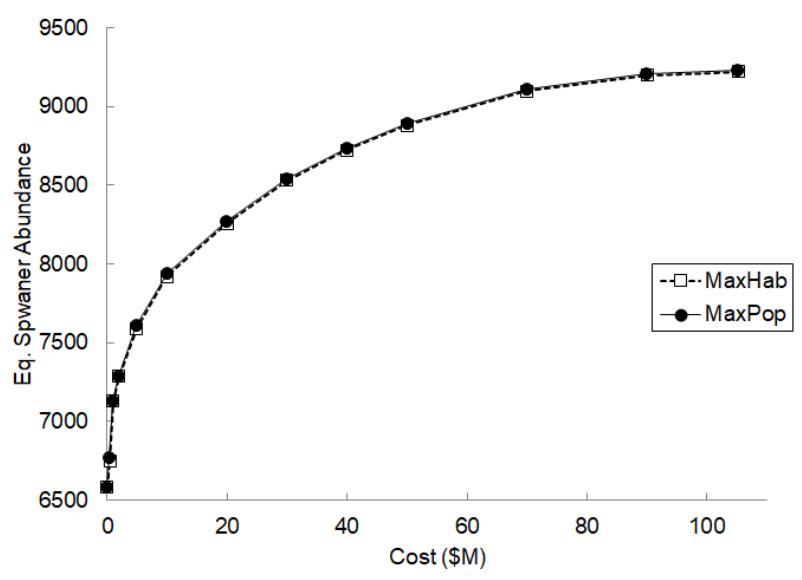

(b)

Figure 5: Accessible spawning habitat (a) and equilibrium spawner abundance (b) versus barrier mitigation cost for MaxPop and MaxHab given reach homing. Note that in both figures, the MaxPop and MaxHab curves nearly overlap for most budgets.

$\mathrm{R}^{2}$ for a Beverton-Holt model was 0.612 , indicating that the Ricker model is a better choice for describing density-dependent growth of wild Tillamook coho. Carrying capacity was subsequently translated to $K=16$ spawners per river $\mathrm{km}$ based on currently accessible spawning habitat. The straying rate was set at $3 \%$, a mid range value for wild coho salmon (Labelle, 1992). As in Newbold and Siikamaki (2009), we used a QET of $10 \%$ of recent (1996-2013) average abundance for wild Tillamook coho salmon, which equated to 615 spawners.

\section{Results}

Results for the MaxPop model are presented in Figures 5 and 6. Accessible habitat and equilibrium population size are plotted against cost of barrier mitigation for both reach and river homing dispersal patterns. For comparison purposes, results are also reported for model MaxHab, which maximizes accessible habitat.

Our results show that homing behavior has a significant impact on optimal barrier mitigation strategies to maximize equilibrium population size. For reach homing (Figure 5), MaxPop essentially produces the same population sizes and amounts of accessible habitat as MaxHab. Under this dispersal pattern, mitigation actions that maximize accessible habitat essentially maximize spawner abundance. The sets of barriers selected for mitigation are nearly identical for both models across all budget scenarios considered (typically sharing $96 \%$ of barriers in common). With river homing (Figure 6), however, MaxPop and MaxHab produce markedly different mitigation strategies. In most cases, MaxPop achieves a given population threshold by removing far fewer barriers, and hence at much lower cost, than MaxHab. For example to reach a population 


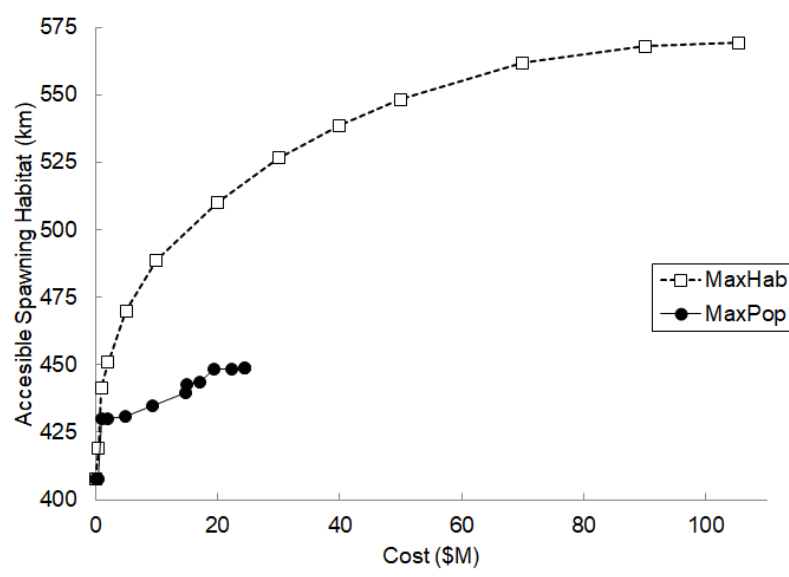

(a)

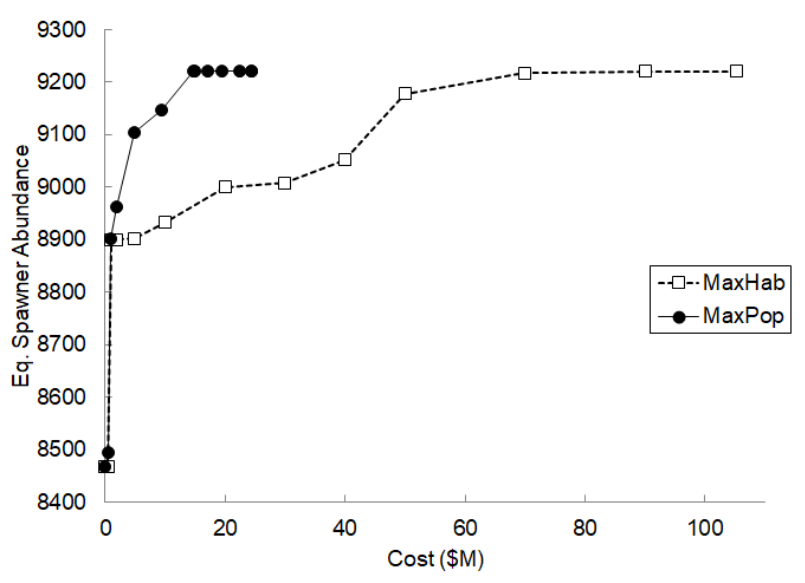

(b)

Figure 6: Accessible spawning habitat (a) and equilibrium spawner abundance (b) versus barrier mitigation cost for MaxPop and MaxHab given river homing.

size of approximately 8900 spawners, 27 barriers would need to be removed at a cost of roughly $\$ 5 \mathrm{M}$ according to MaxHab. In contrast, MaxPop is able to achieve the same spawner abundance by removing only 4 barriers at a cost of around $\$ 1 \mathrm{M}$ (an $80 \%$ cost savings). Similarly, to reach a maximum of 9221 spawners, MaxPop recommends the removal of 37 barriers at a cost of $\$ 14.7 \mathrm{M}$, while MaxHab only achieves this when 182 barriers are removed at a cost of $\$ 90 \mathrm{M}$ (an $84 \%$ cost savings). On the other hand, accessible habitat for MaxPop is substantially lower than it is for MaxHab under a river homing dispersal pattern. For example, accessible habitat for MaxHab reaches a maximum of $569 \mathrm{~km}$ of accessible habitat at a cost of $\$ 105.3 \mathrm{M}$. MaxPop, however, only ever goes up to $449 \mathrm{~km}$ of accessible habitat (21\% less) given $\$ 24.4 \mathrm{M}$.

Mean quasi-extinction probabilities for solutions to MinExP, MaxPop, and MaxHab are provided in Figure 7. In all cases, $95 \%$ confidence intervals were within $\pm 2.8 \%$ of the reported means. The main observation from Figure 7 is the much lower extinction risk achieved by MinExP for any given level of cost in comparison to MaxPop or MaxHab, indicating that additional benefits can be gained by incorporating environmental stochasticity. Without any mitigation action being undertaken, the probability Tillamook coho will reach the quasi-extinction threshold in 50 generations ( 150 years) is $88.4 \%$ for reach homing and $79.9 \%$ for river homing. Extinction probabilities for MinExP rapidly decrease as barrier mitigation resources increase, eventually reaching a minimum of $83.5 \%$ for reach homing at a cost of $\$ 23.2 \mathrm{M}$ and $72.8 \%$ for river homing at a cost of just $\$ 8.8 \mathrm{M}$. Interestingly, MinExP achieves these minimum extinction probabilities by removing only a small subset of barriers - just 43 barriers for reach homing and 18 barriers for river homing that are mostly concentrated low in the basin, as shown in Figure 8.

In comparison, extinction risk initially goes down for both MaxPop and MaxHab but then goes up as barrier 


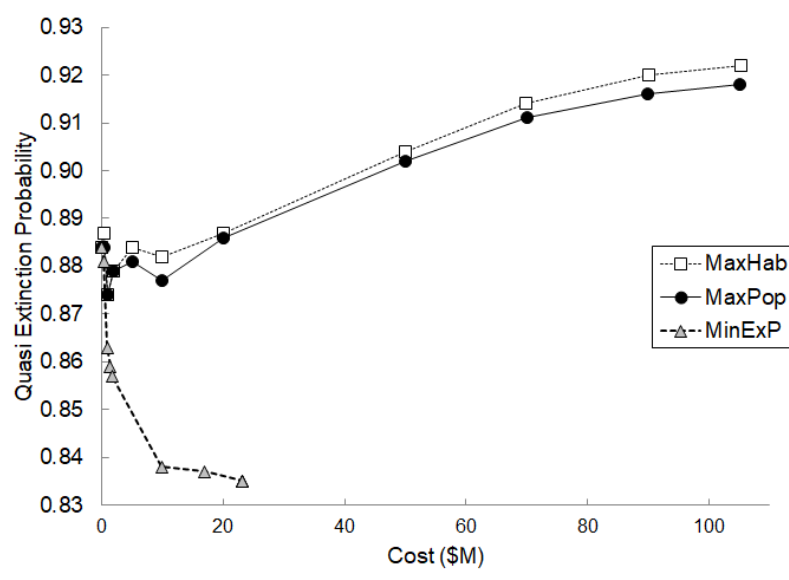

(a)

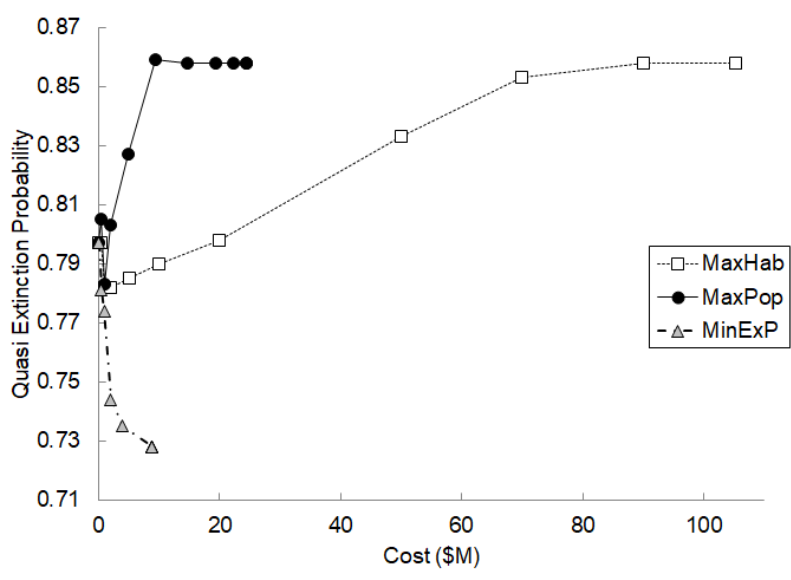

(b)

Figure 7: Mean quasi-extinction probabilities versus barrier mitigation cost for MinExP, MaxPop, and MaxHab given reach homing (a) and river homing (b).

mitigation resources and the number of barriers removed increase, eventually rising above current quasiextinction probabilities. This occurs regardless of the type of homing behavior. When mitigation resources are unrestricted, MaxPop suggests the removal of 191 barriers at a cost of $\$ 105.1 \mathrm{M}$ for reach homing and the removal of 37 barriers at cost $\$ 14.7 \mathrm{M}$ for river homing (see Figure 8). Quasi-extinction risk is not only much higher than MinExP ( $+8.3 \%$ for reach homing and $+13.0 \%$ for river homing), but also higher than under a no-mitigation scenario ( $+3.4 \%$ for reach homing and $+6.2 \%$ for river homing). Removing all 193 barriers at a cost $\$ 105.3 \mathrm{M}$, as recommended by MaxHab, similarly would cause extinction risk to go up $(+3.8 \%$ for reach homing and $+6.1 \%$ for river homing).

As an important side note, we find that equilibrium population size estimates are more heavily influenced by type of homing behavior than are estimates for likelihood of extinction. Moreover, the relative benefits of barrier mitigation differ depending on dispersal pattern and planning goal, with larger increases in fish abundance observed for reach homing and larger decreases in probability of extinction seen with river homing. In particular, for reach homing, MaxPop produces populations sizes ranging from 6580 to 9229 spawners. Here, barrier mitigation yields large gains $(+40 \%)$ in fish abundance. For river homing, population sizes fall in a more narrow range, 8467 to 9221 spawners, with barrier mitigation achieving a more modest improvement in abundance $(+9 \%)$. In contrast, extinction probabilities for MinExP not only vary less between the two homing patterns, but mitigation yields larger reductions in extinction risk if river homing occurs. Specifically, extinction probability decreases from $79.7 \%$ to $72.8 \%$ for river homing (-6.9\%) and from $88.4 \%$ to $83.5 \%$ ($4.9 \%$ ) for reach homing.

In an attempt to provide some high-level guidance regarding optimal barrier selection strategies, we provide 


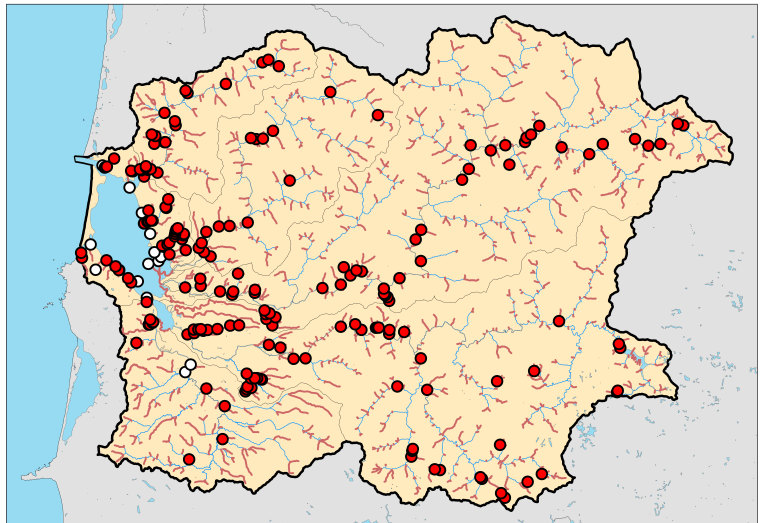

(a)

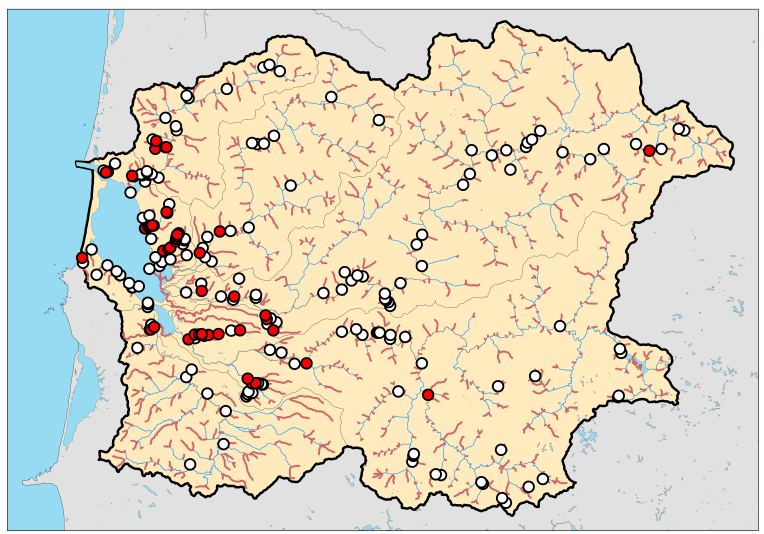

(c)

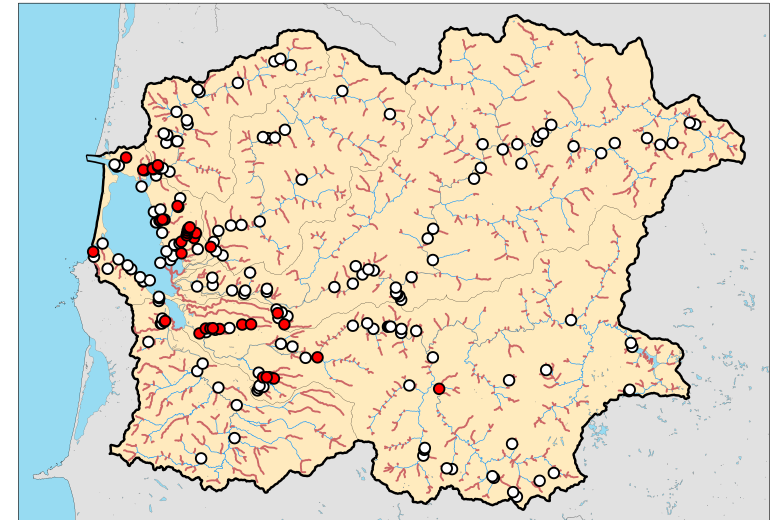

(b)

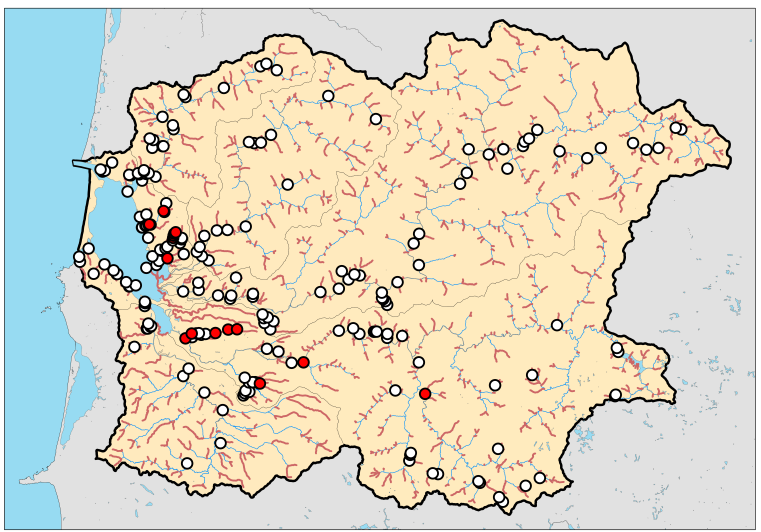

(d)

Figure 8: Barriers targeted for mitigation in the Tillamook basin by MaxPop for reach homing (a) and river homing (b) and by MinExP for reach homing (c) and river homing (d) when mitigation resources are unrestricted. Selected barriers are represented by red circles, unselected barriers by white circles. Spawning areas are indicated by bold, pale red colored river segments. 


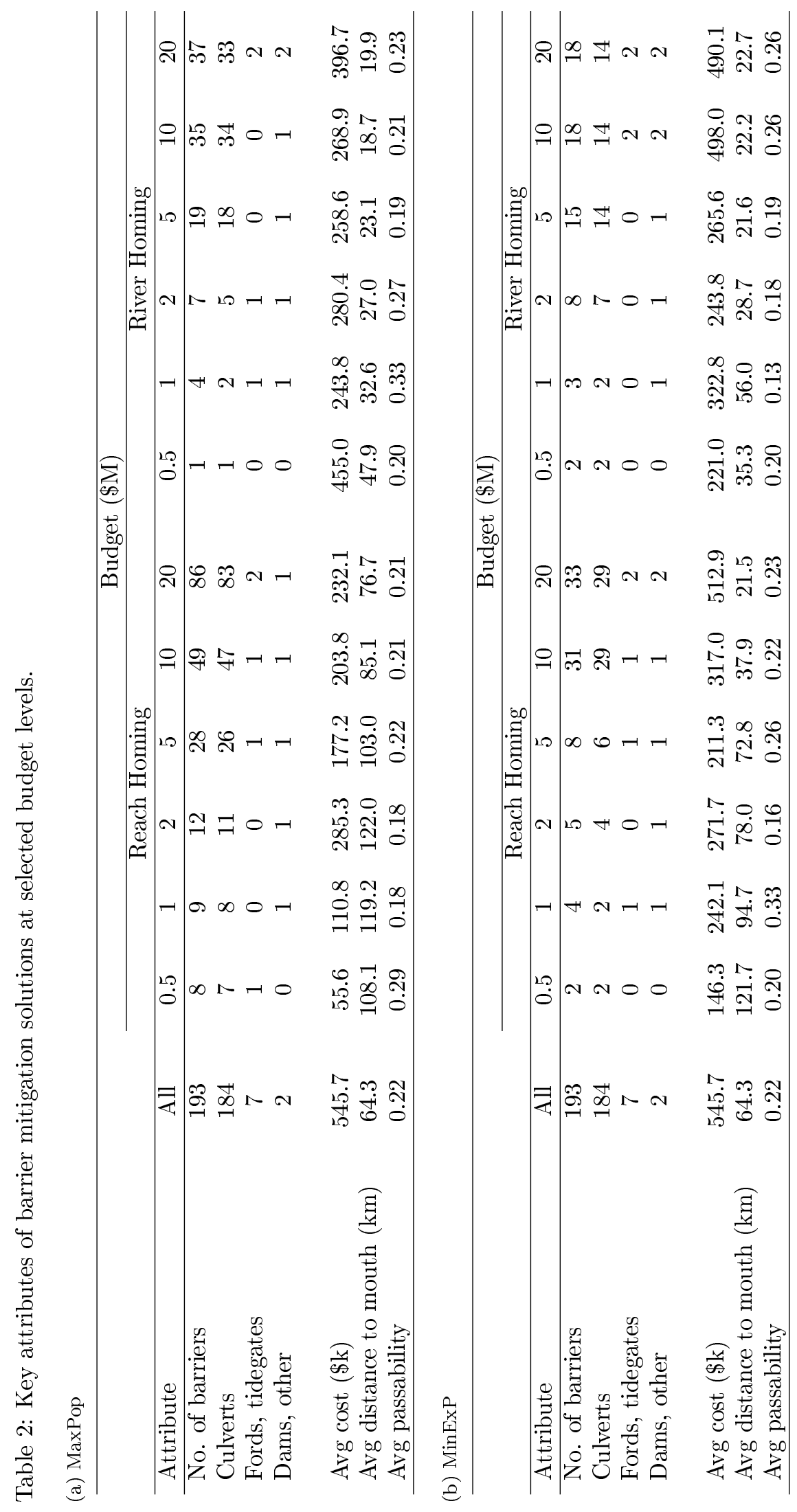


in Table 2 a summary of key attributes of MaxPop and MinExP solutions at selected budget levels. For both MaxPop and MinExP, the spatial distribution of selected barriers differs markedly between the two homing patterns. In particular, barriers selected for mitigation are relatively close to the river mouth for river homing (19-48km on average for MaxPop, 22-56km on average for MinExp) and further away from the river mouth for reach homing (77-122km on average for MaxPop, 22-122km on average for MinExP). Looking at the results in Table 2 more broadly, distance to mouth tends to decrease and average cost per selected barrier increase as the available budget goes up no matter what the planning goal (MaxPop vs MinExP) or homing pattern (reach versus river). In simple terms, when financial resources are scarce (i.e., at low budgets), inexpensive barriers in the upper portions of the basin are preferred, whereas high-cost barriers located nearer to the river mouth are chosen when resources are plentiful (i.e., at high budgets).

\section{Discussion}

The main goal of this study is explore how optimal barrier mitigation strategies are affected by the consideration of fish dispersal and population dynamics. Based on our analysis of wild coho salmon from the Tillamook basin, we find that the choice of homing pattern for spawning adults has a large influence in determining which barriers should be mitigated to maximize equilibrium abundance. With reach homing, near optimal equilibrium population sizes are achieved by simply maximizing accessible habitat. In short, there does not appear to be much benefit from using the more complex and computationally expensive MaxPop model over the simpler MaxHab model. With river homing, however, this is decidedly not the case. For most budget levels, solutions to MaxPop differed markedly from MaxHab. In particular, MaxPop recommends the removal of a much smaller number of barriers in order to maximize spawner population size. What this suggests is that focusing on maximizing accessible habitat may lead to the removal of an excessive numbers of barriers at high cost, while yielding relatively little in terms of increased fish population size. We acknowledge, however, that there may be ancillary benefits from opening up extra river stretches, such as restoring natural flow and sediment patterns or to provide a hedge against the possibility that breeding habitat above barriers or population growth rates have been over estimated.

The inclusion of environmental stochasticity in our analysis produced some very interesting results. Surprisingly, removing all barriers resulted in higher quasi-extinction risk compared to leaving all existing barriers in place regardless of homing pattern. The most straightforward explanation for this is a population thinning effect caused by the presence of fish passage barriers under density dependent population growth. More specifically, depending on the spatial distribution of barriers and spawning habitat, limited amounts of river 
fragmentation can depress spawner densities in certain reaches/subnetworks below carrying capacity, thereby allowing a surplus of recruits to be produced. This, in turn, can help to artificially boost population numbers and improve population persistence in a manner similar to how limited harvesting can potentially increase population growth vis-à-vis the maximum sustainable yield principle (Case, 1999). We emphasize that our results are theoretical, but are supported by Harvey and Railsback (2012), who observed that the largest abundance for a virtual resident trout population occurred at low but positive barrier densities. Similar findings are presented in Labonne et al. (2008), with connectivity having a strong, negative influence on the metapopulation size of a high-dispersal virtual aquatic species.

One of the key benefit of our modeling approach is that it can be readily extended to applications involving a wide variety of migratory fish species, provided that relevant knowledge about dispersal, population growth, and other necessary input data are available. In our analysis, we focused on two extreme forms of homing, reach and river homing, which ostensibly cover the full range of homing fidelity observed among diadromous salmonids. Pacific sockeye salmon (O. nerka), for instance, exhibit the highest degree of homing among salmon, usually returning to their natal incubation sites to spawn (Quinn et al., 1999, 2006). Chinook salmon (O. tshawytscha), on the other hand, tend to home more broadly within their natal stream (Candy and Beacham, 2000; Hamann and Kennedy, 2012). Dispersal for these two species is described fairly accurately by reach and river homing patterns, respectively. There is evidence to suggest that homing for coho salmon (O. kisutch) lies somewhere between these two extremes and is influenced, to some extent, by spawner densities (Anderson and Quinn, 2007). Various degrees of homing are also exhibited by other diadromous (i.e., non-salmonid) fishes. For diadromous fish that home imprecisely (e.g., alewife, Alosa pseudoharengus; shad, Alosinae sp.; and sturgeon, Acipenseridae sp.), one could imagine making suitable adjustments to our proposed dispersal model IFDBP, such that homing occurs within some radius around a natal site and is controlled by density. For diadromous fish that show no discernible homing preference (e.g., eel, Anguilla sp.; and lamprey, Petromyzon and Entosphenus sp.), river homing would be the most suitable choice. Even potadromous fish, which migrate exclusively within a river, could be handled in a relatively straightforward manner, either by making minor modifications to dispersal model IFDBP or relying on the use of dispersal kernels (if dispersal is entirely random).

We emphasize that the results reported here are hypothetical. First, we did not examine how model outputs are affected by uncertainty in key inputs, such as barrier passability values, mitigation costs, habitat estimates, and demographic parameters (e.g., growth rate, carrying capacity, and straying rate). A full-scale sensitivity analysis of these factors, which was beyond the scope of our present work, would be essential before any attempt could be made to apply our models to on-the-ground planning. Second, our findings are based 
a specific form of density dependence, namely the Ricker model. Different models for density dependence (e.g., Beverton-Holt, Gompertz, hockey stick) may produce very different outcomes. Third and perhaps more importantly, we ignore various other factors in our analysis that may be important to long-term population growth and viability, such as juvenile dispersal and survival, demographic stochasticity, Allee effects, hatchery operations, different forms of species interactions (e.g., competition, predation, and parasitism), and straying of coho salmon from or to basins outside the Tillamook. Investigation of any of these aspects would provide interesting lines of future research but would require some ingenuity in terms of devising population models that are computationally efficient enough to be incorporated into an optimization framework.

One of the main take-away messages from our study is that coupling PVA and mathematical optimization can provide tangible benefits to conservation planners. Although PVA-optimization approaches do have higher data requirements and considerably greater computational overhead, which limits their use to well studied species and small- to medium-scale planning problems, where applicable, their greater biological realism has the potential to result in better decision making in terms of developing more effective and efficient conservation strategies. In our particular case, the incorporation of spatiotemporal population dynamics revealed that, at least under certain conditions, strategic removal of a small number of barriers can produce significant fish population gains and that population viability may even be enhanced by leaving some passage barriers in place. The use of simple habitat connectivity index models would invariably fail to capture these sorts of emergent properties and potentially entail wasting limited conservation resources.

\section{Acknowledgments}

C. Ioannidou was supported by a doctoral training grant from the U.K. Engineering and Physical Sciences Research Council (EPSRC). This support is gratefully acknowledged. We also thank three anonymous referees and the associate editor for very helpful comments made on earlier drafts of the paper.

\section{References}

Anderson, J., Quinn, T., 2007. Movements of adult coho salmon (Oncorhynchus kisutch) during colonization of newly accessible habitat. Canadian Journal of Fisheries and Aquatic Sciences 64 (8), 1143-1154.

Bednarek, A. T., 2001. Undamming rivers: a review of the ecological impacts of dam removal. Environmental Management 27 (6), 803-814. 
Candy, J. R., Beacham, T. D., 2000. Patterns of homing and straying in southern british columbia coded-wire tagged chinook salmon (Oncorhynchus tshawytscha) populations. Fisheries Research 47 (1), 41-56.

Case, T. J., 1999. Illustrated guide to theoretical ecology. Ecology 80 (8), 2848-2848.

Eros, T., Schmera, D., Schick, R. S., 2011. Network thinking in riverscape conservation - A graph-based approach. Biological Conservation 144 (1), 184-192.

Fitzpatrick, K. B., Neeson, T. M., 2018. Aligning dam removals and road culvert upgrades boosts conservation return-on-investment. Ecological Modelling 368, 198-204.

Hamann, E. J., Kennedy, B. P., 2012. Juvenile dispersal affects straying behaviors of adults in a migratory population. Ecology 93 (4), 733-740.

Harvey, B., Railsback, S., 2012. Effects of passage barriers on demographics and stability properties of a virtual trout population. River Research and Applications 28 (4), 479-489.

Hornby, D., 2014. RivEX version 10.19 [software]. Available at: http://www.rivex.co.uk, last accessed: 25 Apr 2014.

Januchowski-Hartley, S. R., McIntyre, P. B., Diebel, M., Doran, P. J., Infante, D. M., Joseph, C., Allan, D. J., 2013. Restoring aquatic ecosystem connectivity requires expanding inventories of both dams and road crossings. Frontiers in Ecology and the Environment 11 (4), 211-217.

Kemp, P. S., O'Hanley, J. R., 2010. Procedures for evaluating and prioritising the removal of fish passage barriers: A synthesis. Fisheries Management and Ecology 17 (4), 297-322.

King, S., O’Hanley, J., 2016. Optimal fish passage barrier removal - revisited. River Research and Applications $32(3), 418-428$.

King, S., O'Hanley, J., Newbold, L., Kemp, P., Diebel, M., 2017. A toolkit for optimizing fish passage barrier mitigation actions. Journal of Applied Ecology 54 (2), 599-611.

Kocovsky, P. M., Ross, R. M., Dropkin, D. S., 2009. Prioritizing removal of dams for passage of diadromous fishes on a major river system. River Research and Applications 25 (2), 107-117.

Kuby, M. J., Fagan, W. F., ReVelle, C. S., Graf, W. L., 2005. A multiobjective optimization model for dam removal: An example trading off salmon passage with hydropower and water storage in the Willamette basin. Advances in Water Resources 28 (8), 845-855. 
Labelle, M., 1992. Straying patterns of coho salmon (Oncorhynchus kisutch) stock from southeast Vancouver Island, British Columbia. Canadian Journal of Fisheries and Aquatic Sciences 49 (9), 1843-1855.

Labonne, J., Ravigné, V., Parisi, B., Gaucherel, C., 2008. Linking dendritic network structures to population demogenetics: the downside of connectivity. Oikos 117 (10), 1479-1490.

Lucas, M., Baras, E., 2001. Migration of freshwater fishes. Blackwell Science, Oxford, UK.

Morris, W. F., Doak, D. F., 2002. Quantitative conservation biology: Theory and practice of population viability analysis. Sinauer Associates Inc, Sunderland.

Neeson, T. M., Ferris, M. C., Diebel, M. W., Doran, P. J., O'Hanley, J. R., McIntyre, P. B., 2015. Enhancing ecosystem restoration efficiency through spatial and temporal coordination. Proceedings of the National Academy of Sciences, USA 112 (19), 6236-6241.

Newbold, S. C., Siikamaki, J., 2009. Prioritizing conservation activities using reserve site selection methods and population viability analysis. Ecological Applications 19 (7), 1774-1790.

Nickelson, T. E., Lawson, P. W., 1998. Population viability of coho salmon, Oncorhynchus kisutch, in Oregon coastal basins: Application of a habitat-based life cycle model. Canadian Journal of Fisheries and Aquatic Sciences 55 (11), 2383-2392.

Nieland, J. L., Sheehan, T. F., Saunders, R., 2015. Assessing demographic effects of dams on diadromous fish: A case study for Atlantic salmon in the Penobscot River, Maine. ICES Journal of Marine Science $72(8), 2423-2437$.

NMFS, 2015. Proposed recovery plan for Oregon Coast coho salmon evolutionarily significant unit. Tech. rep., National Marine Fisheries Service, West Coast Region, Portland, Oregon.

NOAA, 2017. Coho salmon. national oceanic and atmospheric administration. Available at: http://www. fisheries.noaa.gov/pr/species/fish/coho-salmon.html, last accessed: 20 Apr 2017.

OASIS, 2016. Oregon Adult Salmonid Inventory and Sampling (OASIS) Project. Oregon Department of Fish and Wildlife. http://odfw.forestry.oregonstate.edu/spawn/index.htm, Last accessed: 23/09/2016.

O'Hanley, J. R., 2011. Open rivers: Barrier removal planning and the restoration of free-flowing rivers. Journal of Environmental Management 92 (12), 3112-3120.

O'Hanley, J. R., Tomberlin, D., 2005. Optimizing the removal of small fish passage barriers. Environmental Modeling and Assessment 10 (2), 85-98. 
O’Hanley, J. R., Wright, J., Diebel, M., Fedora, M. A., Soucy, C. L., 2013. Restoring stream habitat connectivity: A proposed method for prioritizing the removal of resident fish passage barriers. Journal of Environmental Management 125, 19-27.

Paulsen, C. M., Wernstedt, K., 1995. Cost-effectiveness analysis for complex managed hydrosystems: An application to the Columbia River basin. Journal of Environmental Economics and Management 28 (3), $388-400$.

Pilson, S., 2012. Prioritizing fish passage barrier removal at the sub-basin-scale: A strategy for the TillamookNestucca sub-basin, Tillamook County, Oregon. Master's thesis, Portland State University.

Quinn, T., 1997. Homing, straying, and colonization. Genetic effects of straying of nonnative hatchery fish into natural populations. NOAA Tech. Memo. NMFS-NWFSC-30, National Marine Fisheries Service, Seattle.

Quinn, T. P., Stewart, I. J., Boatright, C. P., 2006. Experimental evidence of homing to site of incubation by mature sockeye salmon, Oncorhynchus nerka. Animal Behaviour 72 (4), 941-949.

Quinn, T. P., Volk, E. C., Hendry, A. P., 1999. Natural otolith microstructure patterns reveal precise homing to natal incubation sites by sockeye salmon (Oncorhynchus nerka). Canadian Journal of Zoology 77 (5), $766-775$.

Roni, P., Hanson, K., Beechie, T., 2008. Global review of the physical and biological effectiveness of stream habitat rehabilitation techniques. North American Journal of Fisheries Management 28 (3), 856-890.

Scheuerell, M. D., Hilborn, R., Ruckelshaus, M. H., Bartz, K. K., Lagueux, K. M., Haas, A. D., Rawson, K., 2006. The Shiraz model: A tool for incorporating anthropogenic effects and fish-habitat relationships in conservation planning. Canadian Journal of Fisheries and Aquatic Sciences 63 (7), 1596-1607.

Stanford, J. A., Ward, J., Liss, W. J., Frissell, C. A., Williams, R. N., Lichatowich, J. A., Coutant, C. C., 1996. A general protocol for restoration of regulated rivers. Regulated Rivers: Research \& Management $12(4-5), 391-413$.

Sweka, J. A., Wainwright, T. C., 2014. Use of population viability analysis models for Atlantic and Pacific salmon recovery planning. Reviews in Fish Biology and Fisheries 24 (3), 901-917.

Zheng, P. Q., Hobbs, B. F., 2013. Multiobjective portfolio analysis of dam removals addressing dam safety, fish populations, and cost. Journal of Water Resources Planning and Management 139 (1), 65-75. 